\title{
Evaluation of Complications of Spinal Anesthesia- A Clinical Study
}

\author{
Kapil Lamba ${ }^{1}$ \\ ${ }^{1} \mathrm{HOD}$ \& Professor, Department of Anaesthesia, Rajshree Medical Research Institute \& Hospital Bareilly, Uttar Pradesh.
}

\section{Abstract}

Background: Spinal anaesthesia is one of the most popular and widely used anaesthetic procedures. The present study was conducted to evaluate complications of spinal anesthesia during cesarean section. Subjects and Methods: The present study was conducted in the department of Anesthesiology. It comprised of 78 patients who reported complications in operation theatre during cesarean section. Intraoperative complications such as nausea/ vomiting, hypotension, backache, high spinal block, post-dural puncture headache (PDPH), loss of consciousness, shivering and anesthetic failure was determined. Results: Age group 20-24 years had 28 patients, 24-28 years had 30 and 2832 years had 20 patients. The difference was significant $(\mathrm{P}<0.05)$. The most common complication was shivering seen in 38 , nausea/vomiting in 32 , anesthetic failure in 15, post-dural puncture headache (PDPH) in 14, hypotension in 18, high spinal block in 10, backache in 8 and loss of consciousness in 2. The difference was significant $(\mathrm{P}<0.05)$. Conclusion: Author found that complications of spinal anesthesia vary in different situations. Common complication was shivering, anesthetic failure, Post-dural puncture headache (PDPH), hypotension, nausea, vomiting, high spinal block, backache and loss of consciousness.

Keywords: Complications, Nausea, Spinal anesthesia

Corresponding Author: Dr Kapil Lamba, HOD \& Professor, Department of Anaesthesia, Rajshree Medical Research Institute \& Hospital Bareilly, Uttar Pradesh.

Received: May 2019

Accepted: May 2019

\section{Introduction}

Spinal anaesthesia is one of the most popular and widely used anaesthetic procedures. It is a simple, cost effective and efficient technique that provides complete sensory and motor block, as well as postoperative analgaesia with a high success rate. ${ }^{[1]}$ Several advantages of spinal anaesthesia include a decreased incidence of deep vein thrombosis, reduced intraoperative blood loss, as well as the prevention of pulmonary aspiration in case of emergency, especially in patients with potential airway problems and known respiratory diseases. Anesthetic techniques currently available for caesarean delivery are general and regional anesthesia. Spinal anesthesia for caesarean section is advantageous due to simplicity of technique, rapid administration and onset of anesthesia, reduced risk of systemic toxicity and increased density of spinal anesthetic block. ${ }^{[2]}$

The preference of regional instead of general anesthesia for obstetric surgery is also strongly recommended in the guidelines published by American Society of Anesthesiologists. However, in the presence of any contraindications or emergency state, general anesthesia are mains to be the most common choice for many anesthesiologists. $^{[2]}$ However; general anaesthesia has the advantage of rapid induction, less hypotension, cardiovascular stability and better control over airways and ventilation. ${ }^{[3]}$
At least some of these problems appear to be inevitable and as such, it is not possible to eliminate them all. Fortunately, more severe neurological complications such as death, neuropathy, arachnoiditis and permanent neurologic injury are seldom observed. ${ }^{[4]}$ The present study was conducted to evaluate complications of spinal anesthesia during cesarean section.

\section{Subjects and Methods}

The present study was conducted in the department of Anesthesiology. It comprised of 78 patients who reported complications in operation theatre during cesarean section. The study protocol was approved from institutional ethical committee. Patients were informed regarding the study and written consent was obtained.

General information such as name, age, gender etc. was recorded. Parameters such as heart rate, systolic blood pressure, diastolic blood pressure and respiratory rates were measured at regular interval. Intraoperative complications such as nausea/ vomiting, hypotension, backache, high spinal block, post-dural puncture headache (PDPH), loss of consciousness, shivering and anesthetic failure was determined. Results thus obtained were subjected to statistical analysis. $\mathrm{P}$ value less than 0.05 was considered significant. 


\section{Results}

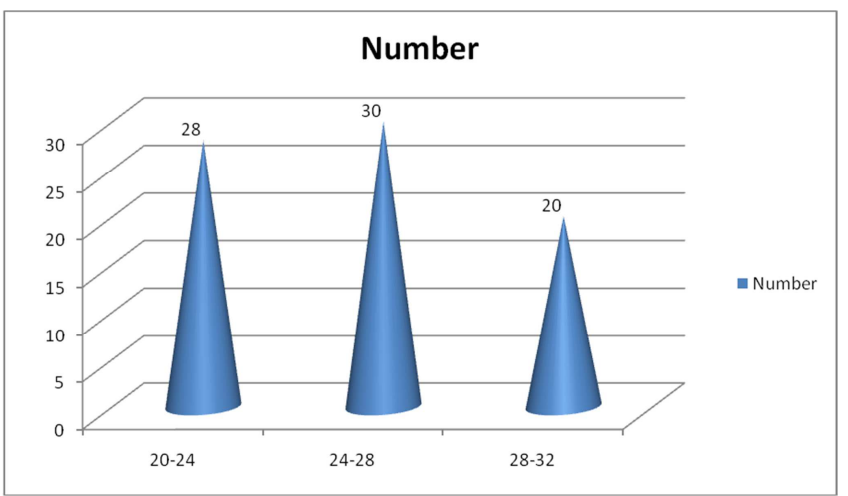

[Table 1] shows that age group 20-24 years had 28 patients, 24-28 years had 30 and $28-32$ years had 20 patients. The difference was significant $(\mathrm{P}<0.05)$.

Table 2: Complications of spinal anesthesia

\begin{tabular}{|l|l|l|}
\hline Complications & Number & P value \\
\hline Nausea/ Vomiting & 32 & \multirow{2}{*}{0.02} \\
\hline Hypotension & 18 & \\
\hline Backache & 8 & \\
\cline { 1 - 2 } High spinal block & 10 \\
\cline { 1 - 2 } $\begin{array}{l}\text { Post-dural puncture headache } \\
\text { (PDPH) }\end{array}$ & 14 & \\
\cline { 1 - 2 } Loss of consciousness & 2 & \\
\hline Shivering & 38 & \\
\cline { 1 - 2 } Anesthetic failure & 15 & \\
\hline
\end{tabular}

[Table 2] shows that most common complication was shivering seen in 38, nausea/vomiting in 32, anesthetic failure in 15 , post-dural puncture headache (PDPH) in 14 , hypotension in 18, high spinal block in 10, backache in 8 and loss of consciousness in 2. The difference was significant $(\mathrm{P}<0.05)$.

\section{Discussion}

Spinal anesthesia is a technique of introducing anesthetic drugs into the subarachnoid space to abolish temporarily the sensory and motor functions of several groups of spinal nerves. Spinal anesthesia recently has gained popularity for cesarean section. Spinal anesthesia has a shorter onset time, but treatment for hypotension is more likely if spinal anesthesia is used. ${ }^{[1]}$ The change in attitude that has taken place is related to an increased awareness that regional block methods are safer for both mother and infant. ${ }^{[5]}$

Spinal anaesthesia in obstetrics differs from spinal anaesthesia in non-pregnant patients in several ways. Smaller doses of local anaesthetic are needed for spinal anaesthesia in pregnancy, and the spread in cerebrospinal fluid (CSF) is less predictable. ${ }^{[6]}$ The present study was conducted to evaluate complications of spinal anesthesia during cesarean section.

In present study, age group 20-24 years had 28 patients, 2428 years had 30 and $28-32$ years had 20 patients. We found that most common complication was shivering seen in 38 , nausea/vomiting in 32 , anesthetic failure in 15 , post-dural puncture headache (PDPH) in 14, hypotension in 18, high spinal block in 10, backache in 8 and loss of consciousness in 2.

Hypotension is an inevitable complication of spinal anaesthesia that occurs when the sympathetic chain becomes blocked, especially when higher dermatome levels are needed. A drop in blood pressure may initiate nausea and vomiting, indicating ischaemia on the spinal cord, which in turn induces an undesired condition for the patient and operating staff. ${ }^{[7]}$

PDPH is a troublesome complication, mostly observed in middle-aged women and the obstetric population. Lower body mass index, previous PDPH and the presence of chronic headaches are other risk factors. Headache rarely occurs in the paediatric population, especially in neonates, but some physicians believe that this may be due to the inability to communicate pain in early childhood. Radicular symptoms, including pain, a burning sensation on the buttocks, dysaesthesia and paraesthesia may be observed following spinal anaesthesia. These symptoms generally subside within two days. ${ }^{[8]}$

Spinal anaesthesia in patients with coexisting infection is a controversial issue. Gritsenko et al, ${ }^{[9]}$ retrospectively reviewed patients who had undergone removal of an infected prosthesis due to hip or knee arthroplasty performed under neuraxial anaesthesia to look for possible associations between perioperative infection and postoperative neuraxial complications regarding meningitis or epidural abscess. Although higher incidence of positive joint culture or pus was found during these procedures, none of the patients included in the 474 cases demonstrated infectious complications during the postoperative period.

Failure of spinal anesthesia is one of the most embarrassing complications for the patient and the anesthesiologist. Spinal anesthesia, in contrast to many other regional anesthesia methods, has a clear end point indicating correct needle placement. Despite this, there is, in common with other regional anesthesia techniques, a potential risk for failure. Correspondingly, even general anesthesia may be associated with failure, as patients can be aware of the surgical operation during anesthesia. Failure rates may be reduced by proper selection of patients, timing, and the skill of the anesthesiologist. $^{[10]}$ The reasons for failure in spinal blocks are in most cases related to technical factors rather than to the anesthetic agent used.

\section{Conclusion}

Author found that complications of spinal anesthesia vary in different situations. Common complication was shivering, anesthetic failure, Post-dural puncture headache (PDPH), hypotension, nausea, vomiting, high spinal block, backache and loss of consciousness.

\section{References}

1. Hebert, et al; complications of spinal anesthesia. An Evaluation of the Complications Encountered in 5,763. Consecutive Spinal Anesthesias. JAMA, J Am Med Assoc. 1950; 142(8):551-557.

2. Cheney FW. A comparison of obstetric and non-obstetric anaesthesia malpractice claims. Anaesthesiology 1991; 74: 242-9.

3. Bucklin BA, Hawkins JL, Anderson JR, Ullrich FA. Obstetric anesthesia workforce survey: twenty-year update. Anesthesiology 2005; 103:645. 
4. Pamela Morgan. Spinal anesthesia in obstetrics. Canadian journal of Anaesthesia 1995; 42: 1145.

5. Carpenter RL. Hyperbaric lidocaine spinal anesthesia: do we need an alternative? Anesth Analg 1995;81:1125-1128.

6. Goodman EJ, DeHorta E, Taguiam JM. Safety of spinal and epidural anesthesia in parturients with chorioamnionitis. Reg Anesth Pain Med. 1996;21:436-41.

7. Sviggum HP, Jacop AK, Arendt KW, Mauermann ML, Horlocker TT, Hebl JR. Neurologic complications after chlorhexidine antisepsis for spinal anesthesia. Reg Anesth Pain Med. 2012;37:139-44.
8. Pollock JE, Neal JM, Stephenson CA, et al. Prospective study of the incidence of transient radicular irritation in patients undergoing spinal anesthesia. Anesthesiology 1996;84: 1361-1367.

9. Gritsenko K, Marcello D, Liguori GA, Jules-Elysèe K, Memtsoudis SG. Meningitis or epidural abscesses after neuraxial block for removal of infected hip or knee prosthesis. Br J Anaesth. 2012;108:485-90.

10. Freedman JM, Li D, Drasner K, et al. Transient neurologic symptoms after spinal anesthesia. An epidemiologic study of 1863 patients. Anesthesiology 1998;89:633-641.

Copyright: () the author(s), publisher. Academia Anesthesiologica Internationalis an Official Publication of "Society for Health Care \& Research Development". It is an open-access article distributed under the terms of the Creative Commons Attribution Non-Commercial License, which permits unrestricted non-commercial use, distribution, and reproduction in any medium, provided the original work is properly cited.

How to cite this article: Lamba K. Evaluation of Complications of Spinal Anesthesia- A Clinical Study. Acad. Anesthesiol. Int. 2019;4(1):109-111.

DOI: dx.doi.org/10.21276/aan.2019.4.1.25

Source of Support: Nil, Conflict of Interest: None declared. 